\title{
SCALE EFFECTS IN THE OPTIMAL DESIGN OF A MICROSTRUCTURED MEDIUM AGAINST BUCKLING
}

\author{
Martin P. Bendsee \\ Mathematical Institute, The Technical University of Denmark, DK-2800 Lyngby, Denmark \\ and \\ Nicolas Triantafyllidis \\ Department of Aerospace Engineering, The University of Michigan, Ann Arbor, MI 48109-2140, \\ U.S.A.
}

(Received 22 November 1988 ; in revised form 9 August 1989)

\begin{abstract}
Certain classes of problems in optimal structural design lead naturally to the introduction of periodic microstructured media as the basis material for the construction of a mechanical element. The unit cell size of these microstructures cannot be arbitrarily small, as suggested by the pertaining optimization analyses up to date, and has to be related to the structure's overall dimensions. One physically important mechanism that provides such microstructure size limitations is elastic buckling.

An analytically tractable model of an infinite periodic rectangular planar frame with axially compressed beams is used to study the optimal buckling loads. For any given design, one can find a critical stress above which buckling instability occurs. In addition one can also find the region in the design space for which the optimal critical mode is a global one, i.e. its characteristic length is much larger than the unit cell size. In this region of the design space one can safely use the homogenized material properties to describe the medium, for they provide all the information needed to predict a global buckling instability. In addition to the detailed parametric study for the model problem investigated here, implications for the optimal design against buckling of more general structures are also briefly discussed.
\end{abstract}

\section{INTRODUCTION}

A very interesting feature of structural optimization is that in certain cases the optimal solution to the problem involves microstructures. Some of the best known examples of this behavior include the compliance optimization of plates under bending, where the optimal solution involves stiffeners, or bars under torsion, where the optimal solution generates a porous material (for further details see the comprehensive review article by Olhoff and Taylor, 1983). Moreover, microstructured optimal solutions are also found in the general shape optimization problem (see for example Kohn and Strang, 1986; Bendsøe and Kikuchi, 1988).

For optimization purposes it is usually sufficient to consider only composites with periodic microstructures (see Kohn, 1988). A consistent way to obtain the macroscopic properties of such periodic materials is by using the theory of homogenization (see Bensousson, et al., 1978 ; Sanchez-Palencia, 1980). For as long as the governing equations are linear and elliptic, the homogenization method predicts the macroscopic properties of the medium based on calculations involving only the solid's fundamental cell (see Boccardo and Marcellini 1976; Bensousson et al., 1978; Kesavan, 1979; Sanchez-Palencia, 1980).

In the majority of the optimization problems investigated so far that lead to microstructures, there is no way of predicting the size of the microstructure relative to the strucure's overall dimensions. The reason is that some fundamental physical phenomena have been omitted from the corresponding models. One such important physical mechanism that places limits on the size of the microstructure, or equivalently on the allowable stress levels, is buckling instability in elastic solids. For example in the optimal plate design problem mentioned before (see Cheng and Olhoff, 1982) the stiffeners cannot be arbitrarily thin for they will buckle under compressive stresses. 
In this work attention is focussed on the optimization of microstructured solids against buckling, with particular emphasis on the implied cell size limitations mentioned above. The difficulty of including the possibility of buckling into the modeling of the problem is that the governing equations are inherently nonlinear. Consequently, as shown by Müller (1987), the results of homogenization theory which are based on calculations involving one unit cell break down and the whole medium has to be considered. The reason for this phenomenon is internal buckling which, as discussed in Triantafyllidis and Maker (1985), breaks the fundamental unit cell symmetry of the structure and leads to more complicated equilibrium paths. As shown in Triantafyllidis and Maker (1985), the standard homogenized model based on one cell cannot predict any of the local instabilities, i.e. instabilities of length scale comparable to the unit cell size. but only the global ones, i.e. the instabilities whose characteristic length is much larger than the unit cell dimension. Moreover, the critical stress levels that trigger the local instabilities can be considerably lower than the ones corresponding to the global instabilities. It should also be mentioned at this point that the issue of optimization of microstructured solids against buckling has also been considered by Mignot, et al. (1980); Suquet (1981) for the case of plates. However, due to the approximations involved, a local buckling instability is impossible in their models which consequently do not exhibit any scale effects.

The model problem to be studied here is that of an infinite periodic plane rectangular beam frame whose members are subjected to compressive axial forces. Depending on geometry and material properties the buckling mode can be either a local one or a global one. Of particular interest is the range of parameters for which the buckling mode is a global one, for in this case the instability can be predicted by studying the homogenized solid. The advantage of this model is that the critical loads corresponding to the local and global buckling modes can be found analytically, thus considerably simplifying the optimization calculations.

For a given mass per unit area of the frame and fixed ratio of applied normal stresses one seeks the optimal design of the unit cell (i.e. beam cross sectional properties and sizes) that maximizes the minimum buckling load. All the optimization calculations reported here were carried out numerically for a wide range of design parameters. It is found that for small densities of the solid and for slender beams the optimal solutions correspond to a local mode while for higher densities or stubby beams the global mode is optimal.

For every set of cell design parameters one can thus establish a dimensionless critical stress per unit cell length ratio above which a buckling instability will occur, thus providing a critical size for the unit cell in the case of given applied stress. Moreover one can also determine the part of the design space for which the global mode is the optimal one against buckling, thus providing a safe region in which homogenization theory results can be employed. In the calculations reported here the aforedescribed boundary in design space between global and local modes is almost independent of the ratio of the applied stresses. This encouraging result suggests that it is perhaps possible to compute relatively simple design requirements that need to be satisfied in order to avoid local buckling when averaged material laws are used in optimizing composites with microstructures.

\section{MODEL DERIVATION}

The structure to be modeled here is the infinite rectangular planar frame shown in Fig. 1a. It consists of horizontal and vertical planar beams, i.e. allowed to deform only in the $x_{1}, x_{2}$ plane, which are welded together at the vertices of an infinite rectangular grid pattern of unit cell dimensions $2 \mathrm{a}_{1} \times 2 a_{2}$.

All the beams in the $x_{x}+$ direction have axial stiffness $E A_{\alpha}$ and bending stiffiness $E I_{\alpha}$ where $E$ is the material's Young modulus, while $A_{x}$ and $I_{x}$ are the cross sectional areas and moments of inertia corresponding to the beams in the $x_{x}$ direction respectively. The structure is deforming under the action of compressive forces $N_{\alpha}$ as shown in Fig. 1a. Assume that 


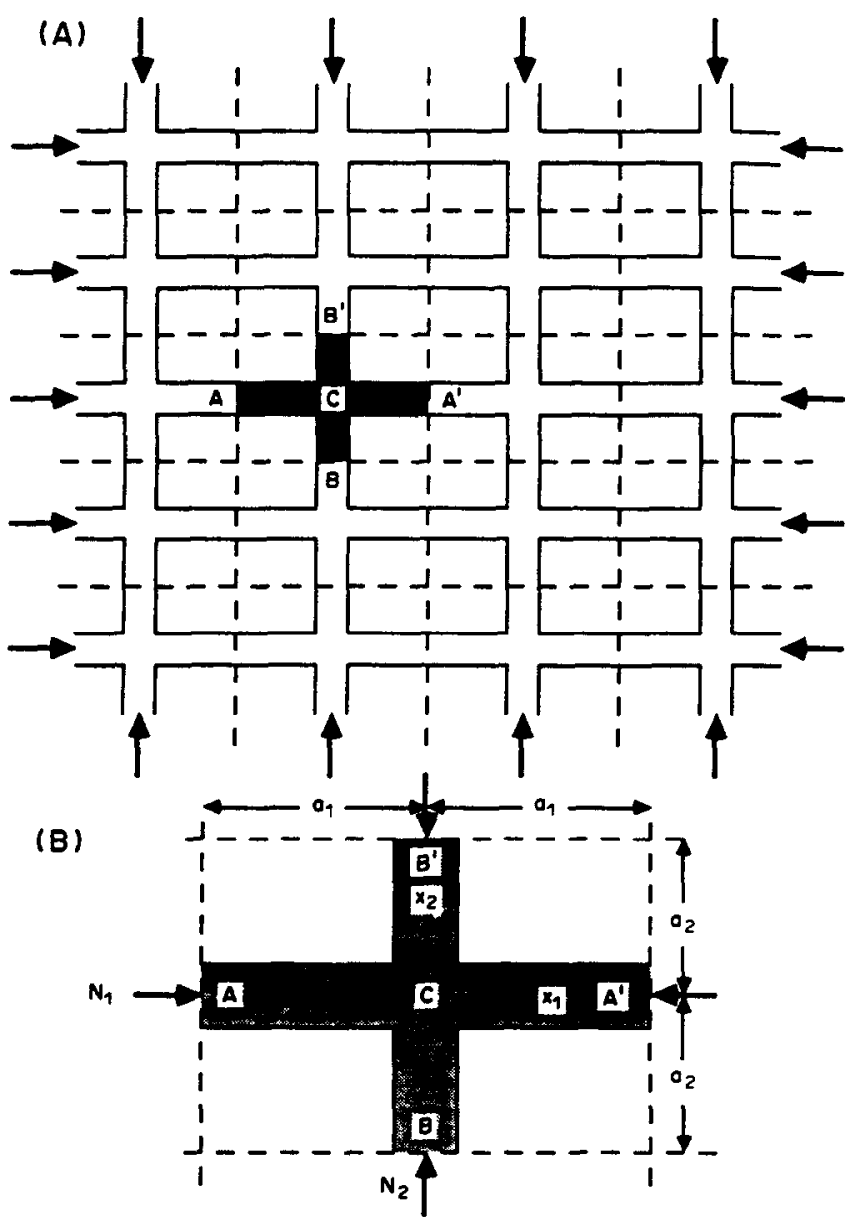

Fig. 1. (A) Schematic diagram of the periodic infinite planar frame. (B) Schematic diagram of the frame's unit cell.

the compressive forces $N_{\alpha}$ are increasing in proportion to a scalar factor $\lambda$, which will be specified in the sequel. For low values of the compressive forces, i.e. for $\lambda$ near zero, the beams remain straight and the corresponding unique solution in which all the beams are loaded axially and have zero moment and shear resultants is termed principal solution.

As $\lambda$ keeps increasing away from zero, it reaches a particular value $\lambda_{c}$, at which a bifurcation buckling instability occurs. Unlike the principal solution, nonzero shear forces and bending moments are developed in the bifurcated equilibrium path and the structure's deformed configuration is no longer rectangular nor (in general) periodic.

The critical load $\lambda_{c}$ can be found as follows. At the onset of bifurcation, incremental equilibrium for the unit cell (see Fig. 1b) implies, using the standard matrix method for nodal force equilibrium (see for example Livesley, 1968), the following relations between the force and displacement increments $\mathbf{f}$ and $\mathbf{d}$ at the five nodes of the cell :

$$
\begin{aligned}
0=\mathbf{f}_{C} & =\left[\left(\mathbf{K}_{11}\right)_{A C}+\left(\mathbf{K}_{11}\right)_{B C}+\left(\mathbf{K}_{11}\right)_{A^{\prime} C}+\left(\mathbf{K}_{11}\right)_{B C}\right] \mathbf{d}_{C} \\
& +\left[\left(\mathbf{K}_{12}\right)_{A C}\right] \mathbf{d}_{A}+\left[\left(\mathbf{K}_{12}\right)_{B C}\right] \mathbf{d}_{B}+\left[\left(\mathbf{K}_{12}\right)_{A^{\prime} C}\right] \mathbf{d}_{A^{\prime}}+\left[\left(\mathbf{K}_{12}\right)_{B C}\right] \mathbf{d}_{B^{\prime}} \\
\mathbf{f}_{A}= & {\left[\left(\mathbf{K}_{21}\right)_{A C}\right] \mathbf{d}_{C}+\left[\left(\mathbf{K}_{22}\right)_{A C}\right] \mathbf{d}_{A} } \\
\mathbf{f}_{B}= & {\left[\left(\mathbf{K}_{21}\right)_{B C}\right] \mathbf{d}_{C}+\left[\left(\mathbf{K}_{22}\right)_{B C}\right] \mathbf{d}_{B} } \\
\mathbf{f}_{A^{\prime}} & =\left[\left(\mathbf{K}_{21}\right)_{A^{\prime} C}\right] \mathbf{d}_{C}+\left[\left(\mathbf{K}_{22}\right)_{A^{\prime} C}\right] \mathbf{d}_{A^{\prime}} \\
\mathbf{f}_{B} & =\left[\left(\mathbf{K}_{21}\right)_{B^{\prime} C}\right] \mathbf{d}_{C}+\left[\left(\mathbf{K}_{22}\right)_{B^{\prime} C}\right] \mathbf{d}_{B^{\prime}}
\end{aligned}
$$


Here the elements of the nodal force vector $f$ are the $x_{1}$ and $x_{2}$ components of the nodal force plus the nodal moment. The corresponding elements of the displacement vector d are the work conjugate components of the nodal displacement plus the nodal rotation. Moreover, $\left(\mathbf{K}_{x \beta}\right)_{e}$ are the incremental stiffnesses corresponding to the beam element $e$, where $e$ stands for $A C, B C, A^{\prime} C, B^{\prime} C$. The indices 1 and 2 correspond to the two ends of the member in question and should not be confused with the corresponding directions in the lattice. The calculation of $\left(\mathbf{K}_{\mathrm{x} \beta}\right)_{e}$ for the structure and the loading in Fig. lb follows a procedure exposed in detail by Livesley (1968) and hence need not be repeated here. For reasons of continuity of the presentation as well as for reference purposes all the necessary formulas for the calculation of $\left(\mathbf{K}_{\alpha \beta}\right)_{e}$ are given in the Appendix (see (A3-A5)).

Note that the infinite frame is a two dimensional elastic structure. Hence at bifurcation it has to satisfy a homogeneous linear equation of the type $\left(L_{\alpha \beta \gamma \delta}\left(\lambda_{c}\right) \dot{u}_{\gamma, \delta}\right)_{, \beta}=0$ (see for example the chapter on stability in Ogden, 1984). Here $L_{x \beta \gamma \delta}\left(\lambda_{c}\right)$ is the structure's incremental moduli tensor evaluated at the onset of bifurcation and $\dot{u}_{x}$ is the corresponding eigenmode. Following Geymonat et al. (1989), one can show that the mode $\dot{u}_{x}\left(x_{1}, x_{2}\right)$ is of the form: $\dot{u}_{\alpha}=\exp \left[i\left(\omega_{1} x_{1}+\omega_{2} x_{2}\right)\right] v_{z}\left(x_{1}, x_{2}\right)$, where $v_{\alpha}\left(x_{1}, x_{2}\right)$ is a periodic function of $x_{1}, x_{2}$ with the same periodicity as that of the structure's. Consequently, for the beam model employed here, one can deduce the following relations for the unit cell at bifurcation:

$$
\begin{aligned}
& \dot{\mathbf{d}}_{A^{\prime}}=\exp \left(2 i \omega_{1} a_{1}\right) \dot{\mathbf{d}}_{A}, \quad \dot{\mathbf{d}}_{B^{\prime}}=\exp \left(2 i \omega_{2} a_{2}\right) \dot{\mathbf{d}}_{B} \\
& \dot{\mathbf{f}}_{A^{\prime}}=-\exp \left(2 i \omega_{1} a_{1}\right) \dot{\mathbf{f}}_{A}, \quad \dot{\mathbf{f}}_{B^{\prime}}=-\exp \left(2 i \omega_{2} a_{2}\right) \dot{\mathbf{f}}_{B} .
\end{aligned}
$$

Introducing (2) into (1) one finds that, for $\left.\left(\omega_{1} a_{1}, \omega_{2} a_{2}\right) \neq 0,0\right)$, a nontrivial solution $\mathbf{d}_{C}$ to (1), (2) exists when:

$\operatorname{det}(\mathbf{K})=0$

$$
\begin{aligned}
\mathbf{K} \equiv & \left(\mathbf{K}_{11}\right)_{A C}+\left(\mathbf{K}_{11}\right)_{A^{\prime} C}+\left(\mathbf{K}_{11}\right)_{B C}+\left(\mathbf{K}_{11}\right)_{B^{\prime} C} \\
& -\left[\mu_{1}\left(\mathbf{K}_{12}\right)_{A^{\prime} C}+\left(\mathbf{K}_{12}\right)_{A C}\right]\left[\left(\mathbf{K}_{22}\right)_{A C}+\left(\mathbf{K}_{22}\right)_{A^{\prime} C}\right]^{-1}\left[\left(\mathbf{K}_{21}\right)_{A^{\prime} C} / \mu_{1}+\left(\mathbf{K}_{21}\right)_{A C}\right] \\
& -\left[\mu_{2}\left(\mathbf{K}_{12}\right)_{B^{\prime} C}+\left(\mathbf{K}_{12}\right)_{B C}\right]\left[\left(\mathbf{K}_{22}\right)_{B C}+\left(\mathbf{K}_{22}\right)_{B^{\prime} C}\right]^{-1}\left[\left(\mathbf{K}_{21}\right)_{B^{\prime} C} / \mu_{2}+\left(\mathbf{K}_{21}\right)_{B C}\right] \\
\mu_{\alpha} \equiv & \exp \left(2 i \omega_{\alpha} a_{\alpha}\right) .
\end{aligned}
$$

The components of the stability matrix $\mathbf{K}$ are found from (3) by substituting the expressions for $\left(\mathbf{K}_{\alpha \beta}\right)_{e}$ in (A3-A5) and subsequently carrying out the required matrix inversions and multiplications. The results are :

$$
\begin{aligned}
& K_{11}=2 E\left[m_{1} a_{1} \sin ^{2}\left(\omega_{1} a_{1}\right)+c_{2}\left(m_{2}\right)^{2} a_{2}\left(F_{2}-B_{2}\right) \sin ^{2}\left(\omega_{2} a_{2}\right)\right] \\
& K_{12}=K_{21}=0 \\
& K_{13}=K_{31}=2 E\left[c_{2}\left(m_{2} a_{2}\right)^{2} F_{2} \sin \left(\omega_{2} a_{2}\right) \cos \left(\omega_{2} a_{2}\right)\right] \\
& K_{22}=2 E\left[m_{2} a_{2} \sin ^{2}\left(\omega_{2} a_{2}\right)+c_{1}\left(m_{1}\right)^{2} a_{1}\left(F_{1}-B_{1}\right) \sin ^{2}\left(\omega_{1} a_{1}\right)\right] \\
& K_{23}=K_{32}=2 E\left[c_{1}\left(m_{1} a_{1}\right)^{2} F_{1} \sin \left(\omega_{1} a_{1}\right) \cos \left(\omega_{1} a_{1}\right)\right] \\
& K_{33}=2 E\left\{c_{1}\left(m_{1}\right)^{2}\left(a_{1}\right)^{3}\left[F_{1}-G_{1} \sin ^{2}\left(\omega_{1} a_{1}\right)\right]+c_{2}\left(m_{2}\right)^{2}\left(a_{2}\right)^{3}\left[F_{2}-G_{2} \sin ^{2}\left(\omega_{2} a_{2}\right)\right]\right\}
\end{aligned}
$$

where for convenience the following notations have been employed:

$$
\begin{aligned}
& m_{\alpha} \equiv A_{\alpha} /\left(a_{\alpha}\right)^{2}, \quad c_{\alpha} \equiv 12 I_{\alpha} /\left(A_{\alpha}\right)^{2}, \\
& F_{\alpha} \equiv\left[\left(\sigma_{\alpha}\right)^{2}-\left(\tau_{\alpha}\right)^{2}\right] / 12 \sigma_{\alpha}, \quad B_{\alpha} \equiv\left(\beta_{\alpha}\right)^{2} / 12=N_{\alpha} / E c_{\alpha}\left(m_{\alpha} a_{\alpha}\right)^{2}, \\
& G_{\alpha} \equiv\left\{\left[\left(\sigma_{\alpha}+\tau_{\alpha}\right)^{2}\right] /\left[2\left(\sigma_{\alpha}+\tau_{\alpha}\right)-\left(\beta_{\alpha}\right)^{2}\right]-\left[\left(\tau_{\alpha}\right)^{2} / \sigma_{\alpha}\right]\right\} / 12 .
\end{aligned}
$$

The functions of $\sigma_{x}\left(\beta_{x}\right)$, and $\tau_{\alpha}\left(\beta_{x}\right)$ are given in the Appendix (see (A4)). 
From (3-5) it is observed that for given dimensionless wave numbers $\omega_{x} a_{x}$ of the bifurcation eigenmode, geometric parameters $a_{x}, m_{x}, c_{x}$ (or equivalently for given dimensions of the unit cell $2 a_{x}$, moments of inertia $I_{x}$ and cross sectional areas $A_{x}$ of the beams) and modulus of elasticity $E$, one can find the axial forces $N_{x}$ at the onset of buckling. The compressive loads $N_{x}$ are assumed to be applied in proportion to a dimensionless load factor $\lambda$ which for convenience is defined by:

$$
\begin{aligned}
\lambda \cos \theta\left[\left(a_{1}\right)^{2}+\left(a_{2}\right)^{2}\right]^{1 / 2} E & =N_{1} / 2 a_{2}, \\
\lambda \sin \theta\left[\left(a_{1}\right)^{2}+\left(a_{2}\right)^{2}\right]^{1 / 2} E & =N_{2} / 2 a_{1} .
\end{aligned}
$$

Here $N_{1} / 2 a_{2}, N_{2} / 2 a_{1}$ represent the average compressive stresses on the frame along the $x_{1}$ and $x_{2}$ directions, respectively. In a loading process the angle $\theta$ (or equivalently the ratio of the average stresses in each direction) is considered fixed while $\lambda$ increases away from zero.

If $\lambda^{*}\left(\omega_{x} a_{x}\right)$ denotes the minimum positive root of (3), then the critical load $\lambda_{c}$ of the structure is the minimum root over all the possible modes, i.e.

$$
\lambda_{c}=\min _{0 \leqslant \omega_{x} a_{x} \leqslant \pi^{\prime} 2}\left[\lambda^{*}\left(\omega_{x} a_{x}\right)\right] \text { where } \operatorname{det}\left[\mathbf{K}\left(\lambda^{*}\right)\right]=0 .
$$

Here only the interval $\omega_{x} a_{x} \in[0, \pi / 2] \times[0, \pi / 2]$ needs to be considered since the form of $\mathbf{K}$ implies that det $[\mathbf{K}]$ is a polynomial in $\sin ^{2}\left(\omega_{z} a_{x}\right) \equiv s_{x}\left(\operatorname{det}[\mathbf{K}]=\Sigma\left[A_{x \beta}(\lambda) s_{z} s_{\beta}\right.\right.$ $\left.+A_{2 \beta_{i} ;}(\lambda) s_{x} s_{\beta} s_{i j}\right]$, where summation is implied for repeated indexes from 1 to 2 . The coefficients $A_{z \beta}(\lambda)$ are given in (8)).

Two typical $\lambda^{*}\left(s_{x}\right)$ surfaces are plotted in Fig. 2. More specifically Fig. 2a gives $\lambda^{*}\left(s_{x}\right)$

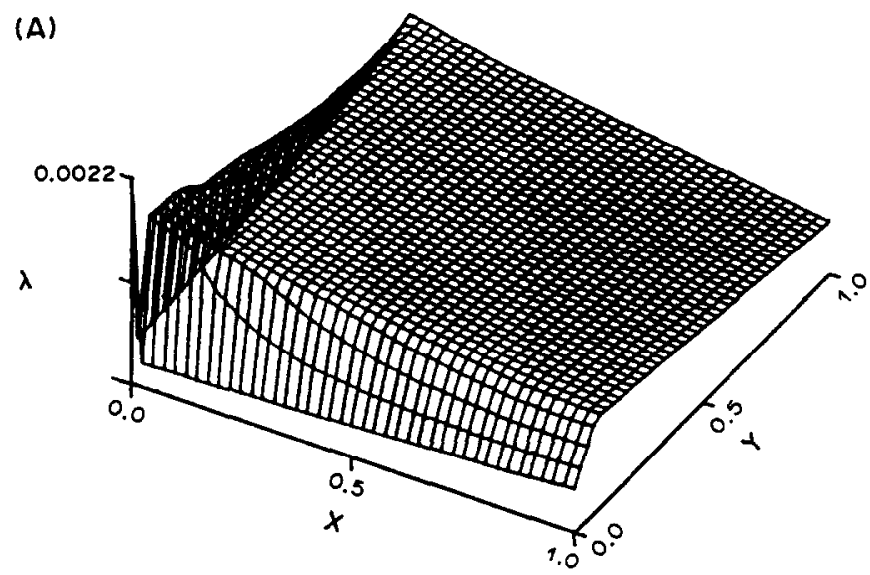

(B)

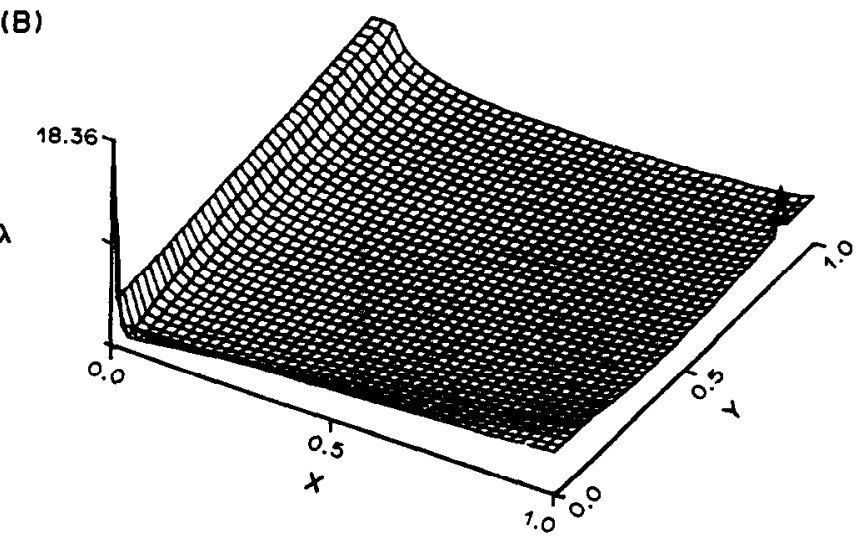

Fig. 2. (A) Typical critical load $\lambda_{z}$ as a function of the mode's wavenumber parameters $s_{x} \equiv \sin ^{2}\left(\omega_{x} a_{x}\right)$ for a frame with slender beams. (B) Typical critical load $\lambda_{c}$ as a function of the mode's wavenumber parameters $s_{x} \equiv \sin ^{2}\left(\omega_{x} a_{z}\right)$ for a frame with stubby beams. 
for relatively slender beams while Fig. $2 b$ shows the surface $\lambda^{*}\left(s_{z}\right)$ for stubby beams. Notice that the critical load $\lambda_{c}$ (the minimum of $\lambda^{*}$ according to (7)) occurs near the origin $s_{1}=s_{2}=0$. This property has been verified numerically for a great number of combinations of the various geometric parameters of the frame and is consequently assumed to be always true. The fact that the surface $\lambda^{*}\left(s_{z}\right)$ is singular at the origin $s_{1}=s_{2}=0$ comes as no surprise, as this point corresponds to two different bifurcation modes: On one hand, observe from (2) that $s_{x}=0$ (or equivalently $\omega_{x} a_{x}=n \pi$ for $n \in \mathbf{Z}$ ) corresponds to a mode that is one cell periodic in the $x_{x}$ direction. On the other hand, by taking the limit $\omega_{z} a_{z} \rightarrow 0$ along different lines in the $\omega_{x} a_{x}$ plane (i.e. assuming that $\omega_{u} a_{x}=\varepsilon \Omega_{x}$ with $\varepsilon \rightarrow 0$ but $\left(\Omega_{1}\right)^{2}+\left(\Omega_{2}^{2}\right)=1$ ) one finds by using (3) and (4), that at the limit $\varepsilon \rightarrow 0, \lambda^{*}\left(\varepsilon \Omega_{x}\right)$ tends to the minimum positive root of :

$$
\begin{gathered}
\left(\Omega_{1}\right)^{4} A_{11}(\lambda)+2\left(\Omega_{1} \Omega_{2}\right)^{2} A_{12}(\lambda)+\left(\Omega_{2}\right)^{4} A_{22}(\lambda)=0 \\
A_{11}(\lambda) \equiv c_{1}\left(m_{1}\right)^{3}\left(a_{1}\right)^{2}\left[-c_{1}\left(m_{1}\right)^{2}\left(a_{1}\right)^{3} F_{1} B_{1}+c_{2}\left(m_{2}\right)^{2}\left(a_{2}\right)^{3} F_{2}\left(F_{1}-B_{1}\right)\right] \\
A_{12}(\lambda) \equiv(1 / 2)\left\{m_{1} m_{2} a_{1} a_{2}\left[c_{1}\left(m_{1}\right)^{2}\left(a_{1}\right)^{3} F_{1}+c_{2}\left(m_{2}\right)^{2}\left(a_{2}\right)^{3} F_{2}\right]\right. \\
\left.-c_{1} c_{2}\left(m_{1} m_{2}\right)^{2} a_{1} a_{2}\left[c_{1}\left(m_{1}\right)^{2}\left(a_{1}\right)^{3} F_{1} B_{1}\left(F_{2}-B_{2}\right)+c_{2}\left(m_{2}\right)^{2}\left(a_{2}\right)^{3} F_{2} B_{2}\left(F_{1}-B_{1}\right)\right]\right\} \\
A_{22}(\lambda) \equiv c_{2}\left(m_{2}\right)^{3}\left(a_{2}\right)^{2}\left[-c_{2}\left(m_{2}\right)^{2}\left(a_{2}\right)^{3} F_{2} B_{2}+c_{1}\left(m_{1}\right)^{2}\left(a_{1}\right)^{3} F_{1}\left(F_{2}-B_{2}\right)\right] .
\end{gathered}
$$

It can be seen, by calculating for the above root of (8) the corresponding eigenmode of $\mathbf{K}\left(\lambda^{*}\right)$, that the resulting beam deformations are long wavelength modes, i.e. modes with characteristic wavelengths $\Lambda_{x}=2 \pi / \omega_{\alpha} \gg 2 a_{z}$ (since $\omega_{x} a_{x} \rightarrow 0$ ), which exceed by far the unit cell size. The coexistence of these two different types of modes at the neighborhood of the origin explains the resulting singularity.

Finding the bifurcation load $\lambda^{*}(0,0)$ at the origin itself requires special care as rigid body modes can appear since for all loads $\mathrm{d}_{c}=[x, y, 0]$ is an eigenvector (with arbitrary $x, y)$ for the corresponding $\mathbf{K}(\lambda)$. In this case it is not difficult to see that $i^{*}(0,0)$ is the solution of $K_{33}=0$ (see (4), (5)) and the corresponding mode is obviously the mode which is one cell periodic with respect to both directions. In all the numerical calculations $\lambda^{*}(0,0)$ is found to be considerably larger than $\lambda_{\text {c }}$.

According to our (numerically validated) assumption, $\lambda_{c}$ is always found near the origin of the $\omega_{x} a_{x}$ plane. Thus $\lambda_{c}$ is the minimum possible root of (8) over all $\Omega_{x}$ with $\left(\Omega_{1}\right)^{2}+\left(\Omega_{2}\right)^{2}=1$. A simple inspection of (8) gives two possible cases: either (i) $\Omega_{1} \Omega_{2}=0$ at $\lambda_{c}$ or (ii) $\Omega_{1} \Omega_{2} \neq 0$ at $\lambda_{c}$.

- When case (i) occurs $\lambda_{c}$ satisfies $A_{11}\left(\lambda_{c}\right)=0$ if $\Omega_{2}=0$, or $A_{22}\left(\lambda_{c}\right)=0$ if $\Omega_{1}=0$.

- When case (ii) occurs $\lambda_{\varepsilon}$ satisfies $A_{11}\left(\lambda_{c}\right) A_{22}\left(\lambda_{c}\right)-\left(A_{12}\left(\lambda_{c}\right)\right)^{2}=0$ with $A_{11}\left(\lambda_{c}\right), A_{22}\left(\lambda_{c}\right)>0$ and $A_{12}\left(\lambda_{c}\right)<0$.

The interpretation of the bifurcation mode for each case is of interest. In case (i), since $\Omega_{\alpha}=0$, the minimum $\lambda$ occurs along one of the $s_{\alpha}$ axes. This means that the eigenmode is one cell periodic with respect to one of the $x_{z}$ axes with corresponding wavelength $\Lambda_{x}=2 a_{\alpha}$, and that it has an infinitely long wavelength in the other direction. For the case (ii), since $\Omega_{\alpha} \neq 0$ but $\omega_{x} a_{x}=\varepsilon \Omega_{x} \rightarrow 0$, the wavelength of the corresponding critical mode is infinitely long in both the $x_{1}$ and $x_{2}$ directions.

Consequently case (i) is identified with the occurrence of a local mode while case (ii) corresponds to a global (long wavelength) mode. At this point one should also remark that had $\lambda_{c}$ occurred at any other point but the origin of the $[0,1] \times[0,1]$ interval in the $\left(s_{1}, s_{2}\right)$ plane, the corresponding mode would have been local in nature as both wavelengths in that case are finite. The fact that for the frame in question the minimum $\lambda^{*}\left(s_{a}\right)$ occurs in one of the corners of the $[0,1] \times[0,1]$ interval of the $\left(s_{1}, s_{2}\right)$ plane is due to the symmetry of the unit cell with respect to the $x_{x}$ axes. For such a symmetric cell, and under the assumption that the critical eigenmode is unique, it can be shown that each $\omega_{z} a_{z}$ has to be either 0 or $\pi / 2$, i.e. $s_{\alpha}=0$ or 1 , and this justifies that $\lambda^{*}$ is minimal at one of the corners of the $[0,1] \times[0,1]$ interval in the $\left(s_{1}, s_{2}\right)$ plane. For an asymmetric unit cell the lowest critical 
mode is most probably local in nature and the $s_{2}$ 's corresponding to $\lambda_{c}$ are in general expected to be interior points of the $[0,1] \times[0,1]$ interval.

\section{FORMULATION OF THE OPTIMIZATION PROBLEM}

In the previous section the governing equations for the buckling of the infinite periodic frame have been established. The next step is to formulate a structural optimization problem for maximizing the critical buckling load of the frame. This problem is stated in the standard $\max / \min$ form:

For fixed $\theta$, find :

$$
\begin{gathered}
\max _{D}\left\{\min _{0 \leqslant S x \leqslant 1}\left[\lambda^{*}\left(s_{x}\right)\right]\right\} \Leftrightarrow \max _{D}\left\{\lambda_{c}\right\} \\
D=\left[a_{1}, a_{2}, A_{1}, A_{2}, I_{1}, I_{2}\right]
\end{gathered}
$$

subject to a constraint on the given mass per unit area, $\rho$.

The solution to the minimization part of the problem, i.e., the determination of $\lambda_{c}$, is already found in the previous section and corresponds either to a local or global buckling mode depending on the values of $\rho, \theta$ and the point of the design space $D$. To simplify the task at hand, the design space is reduced by assuming $A_{1}=A_{2}$. Moreover, by defining the cell aspect ratio $r$ by $r \equiv a_{1} / a_{2}$ and the dimensionless mass per unit area of the frame $\rho$ by (unit density of the beam material is tacitly assumed):

$$
\rho=\left(2 a_{1} A_{1}+2 a_{2} A_{2}\right) /\left\{\left(2 \mathrm{a}_{1}\right)\left(2 a_{2}\right)\left[\left(\mathrm{a}_{1}\right)^{2}+\left(\mathrm{a}_{2}\right)^{2}\right]^{1 / 2}\right\}
$$

one can rewrite the parameters $m_{\alpha}$ in (5) as:

$$
m_{1}=2 \rho\left(1+r^{2}\right)^{1 / 2} / r(1+r), \quad m_{2}=2 \rho r\left(1+r^{2}\right)^{1 / 2} /(1+r) .
$$

In addition one can introduce the angle $\phi$ to be $\tan \phi \equiv I_{2} / I_{1}$. From (5) and in view of the assumption $A_{1}=A_{2}$ one obtains that the beam shape coefficients $c_{x}$ take the form

$$
c_{1}=c \cos \phi, \quad c_{2}=c \sin \phi
$$

where the beam shape coefficient $c \equiv\left(\left(c_{1}\right)^{2}+\left(c_{2}\right)^{2}\right)^{1 / 2}$ will be considered fixed during the optimization process.

Taking into account the parameters introduced above, the optimization problem in (9) can be recast in the following form:

Find :

$$
\begin{gathered}
\max _{\Delta}\left\{\lambda_{c}\right\} \text { for given } \rho>0, c>0 \text { and } \theta \in[0, \pi / 2] \\
\Delta \equiv\{\phi, r \mid 0<\phi<\pi / 2, r>0\}
\end{gathered}
$$

where $\Delta$ is the new design space and $\lambda_{c}$ is the lowest positive root of:

$$
A_{11}(\lambda) A_{22}(\lambda) A(\lambda)=0
$$

$$
\begin{aligned}
& A_{11} \equiv H \cos \phi\left[-F_{1} B_{1} \cos \phi+r F_{2}\left(F_{1}-B_{1}\right) \sin \phi\right] \\
& A_{12} \equiv\left\{F_{1} \cos \phi+r F_{2} \sin \phi-H^{2} \sin \phi \cos \phi\left[F_{1} B_{1}\left(F_{2}-B_{2}\right) \cos \phi+r F_{2} B_{2}\left(F_{1}-B_{1}\right) \sin \phi\right]\right\} / 2 \\
& A_{22} \equiv H \sin \phi\left[-r F_{2} B_{2} \sin \phi+F_{1}\left(F_{2}-B_{2}\right) \cos \phi\right] \\
& A \equiv\left(A_{12}\right)^{2}-A_{11} A_{22}, \quad H \equiv 2 \rho c\left[\left(1+r^{2}\right)^{1 / 2}\right] /(1+r) .
\end{aligned}
$$

In the expressions above $F_{\alpha}$ are still given in terms of $\sigma_{x}\left(\beta_{x}\right), \tau_{\alpha}\left(\beta_{x}\right)$ by (5), while in 
view of (6), (11), (12), $B_{x}$ in (5) take the form:

$$
B_{1}=\lambda \cos \theta(1+r) / H \rho \cos \phi, \quad B_{2}=\lambda \sin \theta(1+1, r) / H \rho \sin \phi .
$$

Finally, $\sigma_{x}\left(\beta_{x}\right), \tau_{x}\left(\beta_{x}\right)$ are given in terms of $\beta_{x}$ by (A4) while $\beta_{x}$ are still given in terms of $B_{z}$ by (5).

The optimization problem is thus completely specified by (13)-(15). If for a given mass per unit area $\rho$, given beam shape parameter $c$ and given load angle $\theta$, the optimal buckling load $\lambda_{c}$ satisfies $A_{11}\left(\lambda_{c}\right)=0$ or $A_{22}\left(\lambda_{c}\right)=0$, then according to the discussion of Section 2, the critical mode is local in nature. If on the other hand, $A\left(\lambda_{c}\right)=0$ (assuming of course $\left.A_{12}\left(\lambda_{c}\right)<0\right)$ the corresponding critical mode is global in nature.

\section{COMPUTATIONAL RESULTS}

It is noted that the symmetry properties of planar frame model imply that only loading angles $\theta$ between 0 and $\pi / 4$ need to be considered, as designs for $\pi / 4 \leqslant \theta \leqslant \pi, 2$ are covered by a switching of axes. The optimal design for a loading angle $\pi / 2-\theta(\theta \in[0, \pi / 4])$ is then given by $\left(1 / r^{\mathrm{op}}, \pi / 2-\varphi^{\mathrm{op}}\right)$, where $\left(r^{\mathrm{op}}, \varphi^{\mathrm{op}}\right)$ is the optimial design for the loading angle $\theta$, while the corresponding modes are obtained by symmetry with respect to the $x_{1}=x_{2}$ line.

The optimization of buckling loads is complicated by the fact that the smallest positive root of a nonlinear function may have nondifferentiable and even discontinuous dependence on parameters in the function. For linear, elliptic eigenvalue problems the smallest eigenvalue is differentiable when the root is simple and the eigenvalue has generalized gradients when it is multiple (see Haug, et al., 1986). Numerical experiments indicate that $\lambda_{\text {loc }}$, the local buckling load for the present problem (i.e. the lowest positive root of $A_{11}(\lambda)=0$, see (14)) is differentiable as a function of $r$ and $\varphi$, while the global buckling load $\lambda_{\text {glo }}$ (i.e. the lowest positive root of $A(\lambda)=0$ for which also $A_{12}\left(\lambda_{)}<0\right.$, see (14)) is discontinuous at some values of $r$ and $\varphi$. However, the critical load, i.e. the minimum of the local and global buckling loads, is found to be continuous and has subgradients at points of nondifferentiability. These properties mean that the optimization problem formulated in (13) can conveniently be solved by a sequential quadratic approximation algorithm.

The results presented here are computed by employing the program MINCF developed by Madsen and Tingleff (1986). Some typical results from the dependence of the critical load $\lambda_{c}$ on the aspect ratio $r$ calculated for different values of the mass per unit area $\rho$ and the beam shape parameter $\phi$ (and for fixed values of the beam shape parameter $c$ and the load angle $\theta)$ are depicted in Fig. 3. In Fig. 3a ( $\phi=0.4842 \mathrm{rad})$ the beams in the $x_{1}$ direction are stiffer, in Fig. $3 \mathrm{~b}(\phi=0.7842 \mathrm{rad})$ the beams in the $x_{1}$ and $x_{2}$ direction are identical and in Fig. 3c ( $\phi=1.0842 \mathrm{rad})$ the beams in the $x_{1}$ direction are weaker. In all cases the critical load is found to depend continuously on the design parameters. Additional calculations, not shown here, gave similar results for the critical load as a function of the parameter $\phi$. However, it is interesting to note that, although the local buckling load $i_{100}$ is always a continuous function of the design parameters, this is not generally true for the global buckling mode $\lambda_{\mathrm{glo}}$.

The dependence of the local and global critical load on the aspect ratio $r$ for different mass per unit area $\rho$ (and for fixed values of the beam shape parameters $c, \phi$ and the load angle $\theta)$ is depicted in Fig. 4. The lowest mass per unit area $(\log \rho=-1.00)$ corresponds to Fig. $4 \mathrm{a}$, the intermediate mass per unit area $(\log \rho=-0.54)$ corresponds to Fig. $4 \mathrm{~b}$, while the highest mass per unit area $(\log r=-0.35)$ corresponds to Fig. 4c. Note from Fig. $4 \mathrm{a}$ and Fig. $4 \mathrm{~b}$ that for the lower mass per unit area the global buckling load is a discontinuous function of the cell aspect ratio. For a mass per unit area high enough as to ensure that the global load is the critical one the situation reverses itself as one can see in Fig. 4c. Notice that as expected in all cases the critical load is a continuous function of $r$ thus justifying the numerical algorithm adopted for the solution of the optimization problem.

It turned out that the buckling optimization problem treated here is of such a nature 

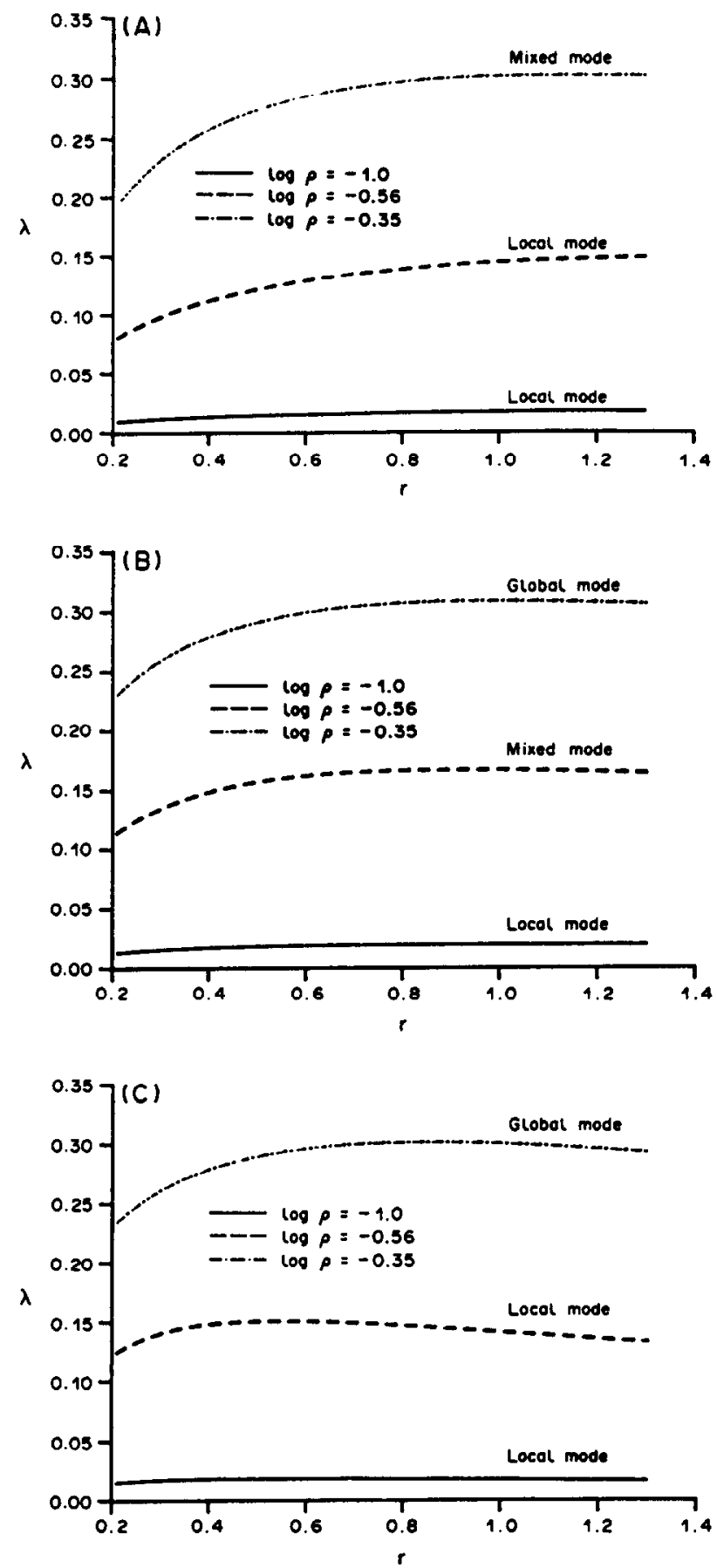

Fig. 3. (A) Critical load $\lambda_{c}$ as a function of the cell aspect ratio $r$ for different mass per unit area parameters $\rho$. Results calculated for $c=30, \phi=0.4842, \theta=0.5236$. (B) Critical load $\lambda_{c}$ as a function of the cell aspect ratio $r$ for different mass per unit area parameters $\rho$. Results calculated for $c=30, \phi=0.7842, \theta=0.5236$. (C) Critical load $\lambda_{c}$ as a function of the cell aspect ratio $r$ for different mass per unit area parameters $\rho$. Results calculated for $c=30, \phi=1.0842, \theta=0.5236$.

that multimodality generically does not occur. Thus, one can find a curve in the $c-\rho$ plane which separates the sets of beam shape parameters $c$ and densities $\rho$ that correspond to local modes and global modes. These curves are of particular interest and they will be discussed subsequently in more detail.

The computer time used for the parametric study of the optimization problem (13) can be reduced considerably by noting that for the local buckling load $\lambda_{\text {loc }}$, the quantity 

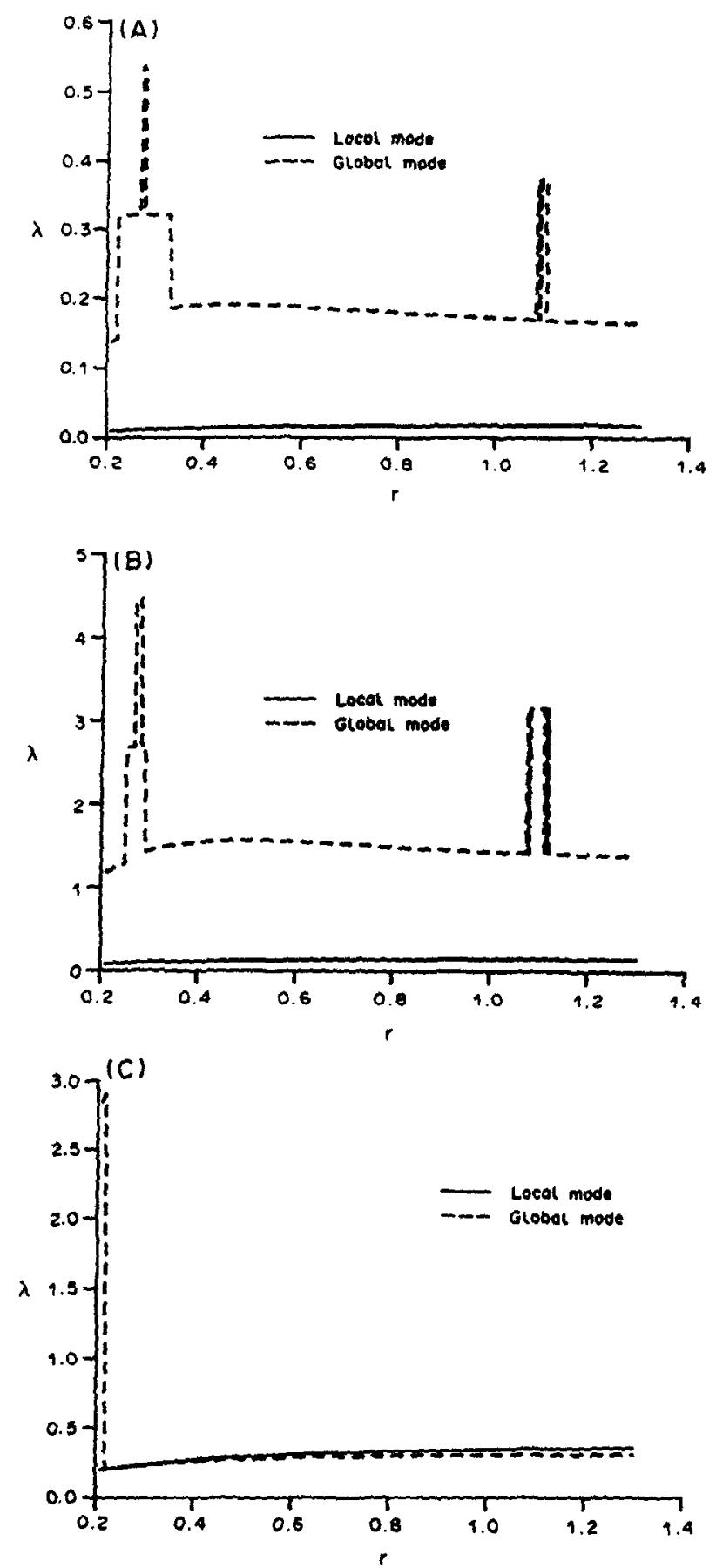

Fig. 4. (A) Local $\lambda_{\text {loc }}$ and global $\lambda_{\text {glo }}$ buckling loads as functions of the cell aspect ratio $r$. Results calculated for $\rho=\exp (-1.00) c=30, \phi=0.7842, \theta=0.5236$. (B) Local $\lambda_{\text {toc }}$ and global $\lambda_{\text {tho }}$ buckling loads as functions of the cell aspect ratio r. Results calculated for $\rho=\exp (-0.54) c=30$, $\phi=0.7842, \theta=0.5236$. (C) Local $\lambda_{1 \alpha}$ and global $\lambda_{\text {vlo }}$ buckling loads as functions of the cell aspect ratio $r$. Results calculated for $\rho=\exp (-0.35) c=30, \phi=0.7842, \theta=0.5236$.

$\lambda_{\text {loc }} / c \rho^{2}$ is a constant, say $\Gamma_{10 c}$, independent of $c$ and $\rho$. Thus, the optimal local root $\lambda_{\mathrm{boc}}^{0 p \text { and }}$ hence $\Gamma_{\mathrm{k}}^{\mathrm{op}}$ is computed for one choice of $c$ and $\rho$, with optimal design $r_{\mathrm{loc}}^{\mathrm{op}}, \varphi_{\mathrm{oc}}^{\mathrm{op}}$. From this, the optimal local buckling load $\lambda_{10}^{0 p}$ is given by $\lambda_{j o c}^{o p}=c \rho^{2} \Gamma_{10}^{o p}$ for all values $c$ and $\rho$ while the corresponding optimal design is the same for all values of $c$ and $\rho$. This information can then be used to reduce the number of times the minimization problem (13) has to be solved. For given values of $c$ and $\rho$ the global buckling load $\lambda_{\text {glo }}$ is computed for the optimal 
design parameters $r_{\mathrm{loc}}^{\mathrm{pp}}, \varphi_{\mathrm{loc}}^{\mathrm{op}}$ and if $\lambda_{\mathrm{loc}}^{\mathrm{pp}} \leqslant \lambda_{\mathrm{glo}}$ one immediately ensures that the solution to (13) is given by $\left(\lambda_{\mathrm{loc}}^{\mathrm{p}}, r_{\mathrm{loc}}^{\mathrm{op}}, \varphi_{\mathrm{lo}}^{\mathrm{op}}\right)$. If this inequality is not satisfied for the specific choice of $c$ and $\rho$, the global buckling load is the critical one and (13) has to be solved.

The derivative information needed for the iterative solution procedure used to solve the optimization problem (13) was obtained by numerical differentiation. This requires a high precision in the computation of the roots of $A_{11}(\lambda), A_{22}(\lambda)$ and $A(\lambda)$, and hence it is very time consuming. However, in view of the algebraic complexity of the aforementioned quantities in terms of the design variables (see (14), (15)), the straightforward numerical differentiation seems to be the only viable method.

The roots of $A_{11}(\lambda), A_{22}(\lambda)$ and $A(\lambda)$ are computed by direct search followed by a bisection method. For every value of $c$ and $\rho$ the optimization is carried out using several different starting points for the iterative procedure. Interestingly, all starting points give rise to the same optimal designs indicating the existence of only one local maximum which
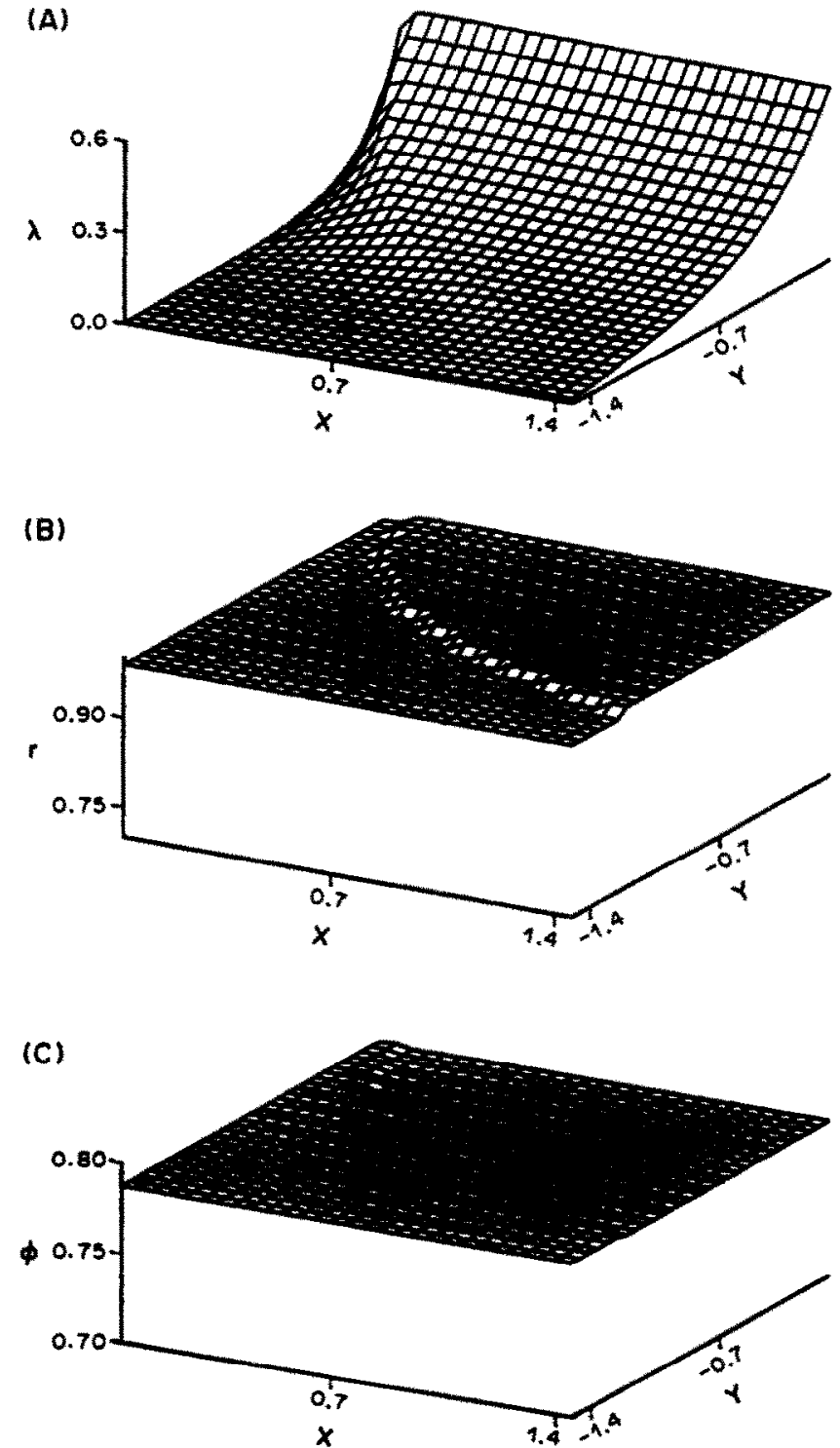

Fig. 5. (A) Optimal critical load $\lambda_{c}$ as a function of the mass per unit area $(y=\log \rho)$ and the beam shape parameter $(x=c / 100)$ for a load angle $\theta=0.7854$. (B) Optimal aspect ratio $r$ as a function of the mass per unit area $(y=\log \rho)$ and the beam shape parameter $(x=c / 100)$ for a load angle $\theta=0.7854$. (C) Optimal beam shape parameter $\phi$ as a function of the mass per area $(y=\log p)$ and the beam shape parameter $(x=c / 100)$ for a load angle $\theta=0.7854$. 
is also the global maximum. The stopping criteria for the optimization is either a requirement that the Kuhn-Tucker conditions for an optimum are satisfied to a certain precision or that the relative improvement in the objective is below some low value $\left(\leqslant 10^{-6}\right)$. It turns out that the optima for the problem are "flat", so that it is extremely time consuming to obtain very high precision in the determination of the resulting optimal designs. This insensitivity of the optimal load on the values of $r$ and $\phi$ explains the "asperities" of the corresponding optimal surfaces (see Figs. $6 \mathrm{~b}-8 \mathrm{~b}$ and Figs $6 \mathrm{c}-8 \mathrm{c}$ ).

The results of the parametric study are shown in Figs 5-8, where optimal critical loads $\lambda_{c}$ and corresponding designs $r$ and $\varphi$ are shown as functions of the beam shape parameter $c$ and the mass per unit area $\rho$. More specifically Figs 5-8, give the optimal solution of the frame buckling problem for load angles $\theta=\pi / 4, \theta=\pi / 6, \theta=\pi / 12$ and $\theta=0$ respectively; parts $A, B$ and $C$ of each one of these figures depict the dependence of the optimum critical load $\lambda_{\mathrm{s}}$, the optimal cell aspect ratio $r$ and the optimal beam shape parameter $\phi$ as functions of the mass per unit area parameter $\rho$ and the beam shape parameter $c$. Note that for very

(A)

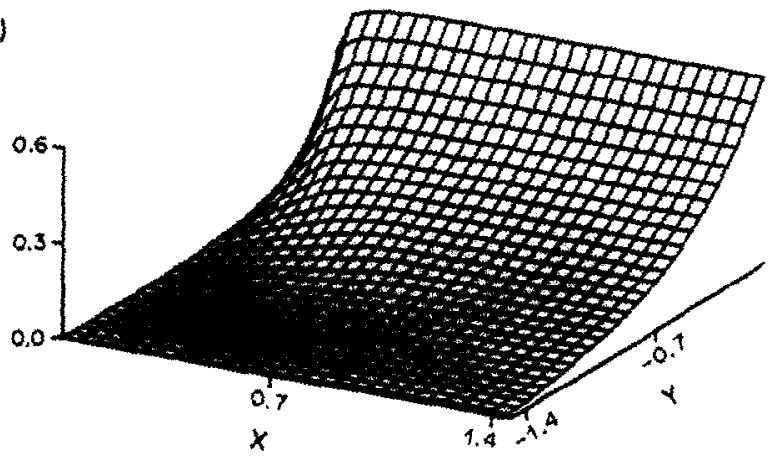

(8)

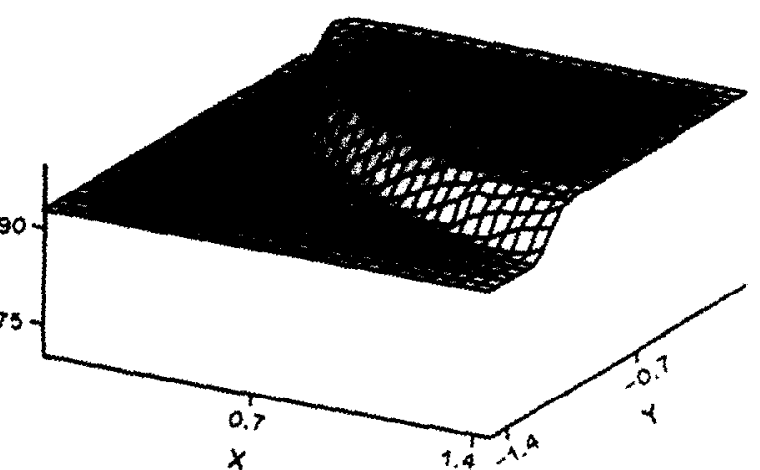

(c)

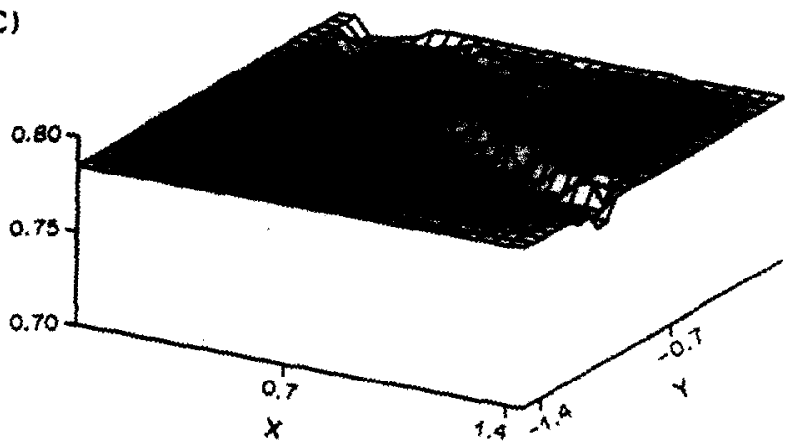

Fig. 6. (A) Optimal critical load $\lambda_{c}$ as a function of the mass per unit arta $(y=\log \rho)$ and the beam shape parameter $(x=c / 100)$ for a load angle $\theta=0.5236$. (B) Optimal aspect ratio $r$ as a function of the mass per unit area $(y=\log \rho)$ and the beam shape parameter $(x=c / 100)$ for a load angle $\theta=0.5236$. (C) Optimal beam shape parameter $\phi$ as a function of the mass per unit area $(\lambda=\log \rho)$ and the beam shape parameter $(x=c / 100)$ for a load angle $\theta=0.5236$ 
stubby beams (i.e. for high densities $\rho$ or high beam shape parameters $c$ ) the optimal critical load corresponds to a global mode. For slender beams (i.e. for low densities $\rho$ and low beam shape parameters $c$ ) the optimal design corresponds to a local mode. It is interesting to note that for all loading angles and all parameters $c$ and $\rho$, the optimal design is not far from the completely symmetric design of $r=1$ and $\varphi=\pi / 4$, while the most unsymmetrical designs are obtained for a loading angle $\theta=0$, as is to be expected. Obviously the flat part of the $r$ versus $c, \rho$ and the $\phi$ versus $c, \rho$ surfaces in parts $B$ and $C$ of these figures corresponds to the case where the local mode is the optimal one as it has been previously explained.

The values of the beam shape parameter $c$ and the density $\rho$ for which the optimum critical buckling solution shifts from local to global modes are shown in Fig. 9. Notice that this boundary is surprisingly insensitive to variations of the loading ratio angle $\theta$. Most important, the curves in Fig. 9 in conjunction with Figs. 5a-8a contain the sought after size effect of buckling on the microstructure. Since the dimensionless critical load $\lambda_{c}$ is by
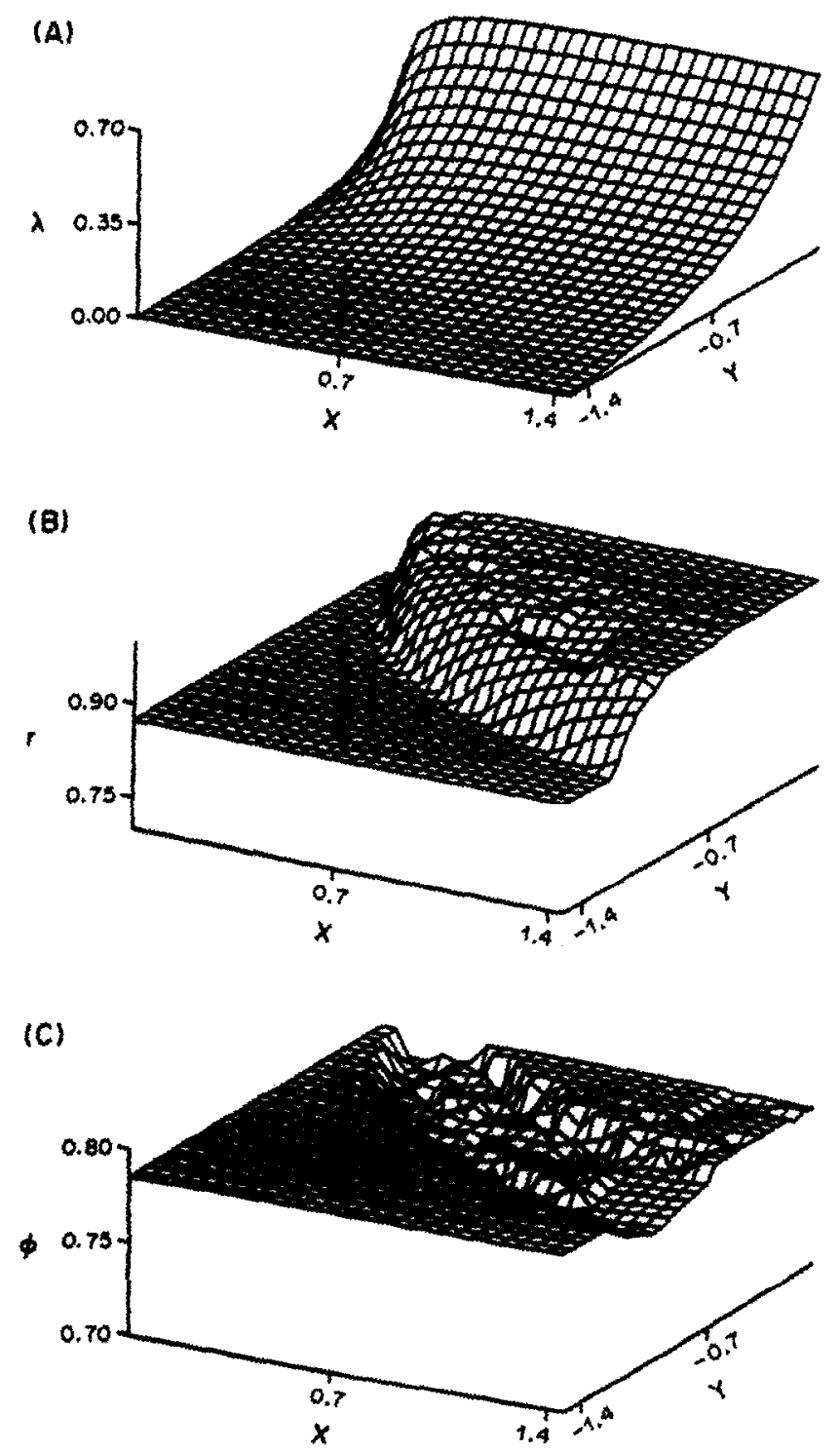

Fig. 7. (A) Optimal critical load $\lambda_{c}$ as a function of the mass per unit area $(y=\log \rho)$ and the beam shape parameter $(x=c / 100)$ for a load angle $\theta=0.2618$. (B) Optimal aspect ratio $r$ as a function of the mass per unit area $(y=\log \rho)$ and the beam shape parameter $(x=c / 100)$ for a load angle $\theta=0.2618$. (C) Optimal beam shape parameter $\phi$ as a function of the mass per unit area $(y=\log \rho$ ) and the beam shape parameter $(x=c(100)$ for a load angle $\theta=0.2618$. 
definition (see (6)) a critical stress, as a fraction of the Young's modulus $E$, divided by half the unit cell diagonal $\left[\left(a_{1}\right)^{2}+\left(a_{2}\right)^{2}\right]^{1 / 2}$ one determines from the surfaces in Figs $5 \mathrm{a}-8 \mathrm{a}$ the critical unit cell dimensions below which buckling will occur for a given stress level. In addition the curves in Fig. 9 determine the critical unit cell parameters below which a local buckling will occur for a given stress level.

Hence, for a given unit cell size, a standard homogenization theory approach can be safely used to describe the properties of this medium only for dimensionless stress levels remaining below the surface of optimal $\lambda_{c}$ for the corresponding values of $\rho$ and $c$. Moreover, if for the cell size in question the dimensionless density $\rho$ and corresponding beam shape parameter $c$ are in the global mode region of Fig. 9 then a homogenized model can be employed irrespective of the applied stress levels since it can predict the global instability as discussed in Triantafyllidis and Maker (1985).
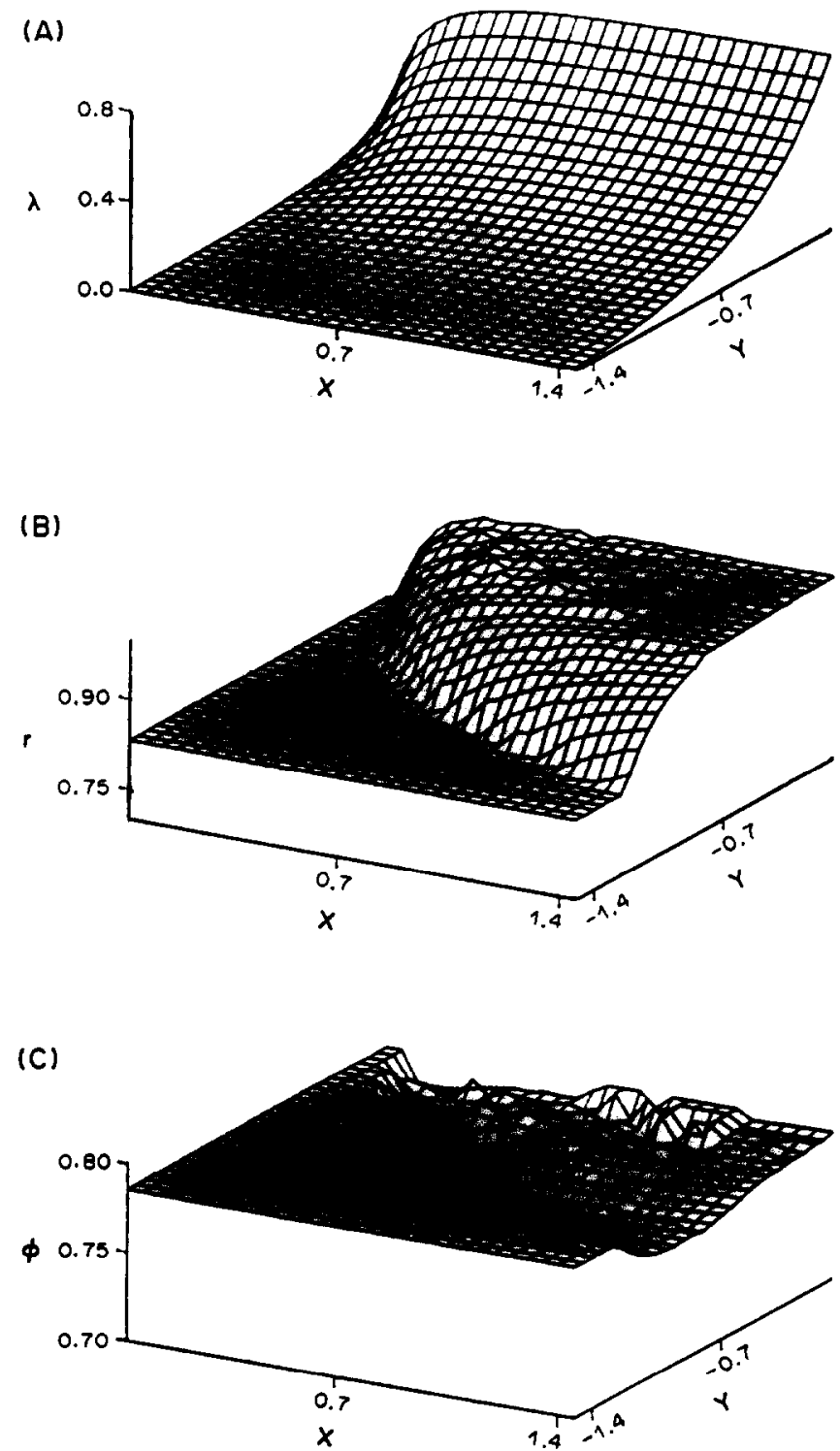

Fig. 8. (A) Optimal critical load $\lambda_{c}$ as a function of the mass per unit area $(y=\log \rho)$ and the beam shape parameter $(x=c / 100)$ for a load angle $\theta=0$. (B) Optimal aspect ratio $r$ as a function of the mass per unit area $(y=\log \rho)$ and the beam shape parameter $(x=c / 100)$ for a load angle $\theta=0$. (C) Optimal beam shape parameter $\phi$ as a function of the mass per unit area $(y=\log \rho)$ and the beam shape parameter $\left(x=c_{;} 100\right)$ for a load angle $\theta=0$. 


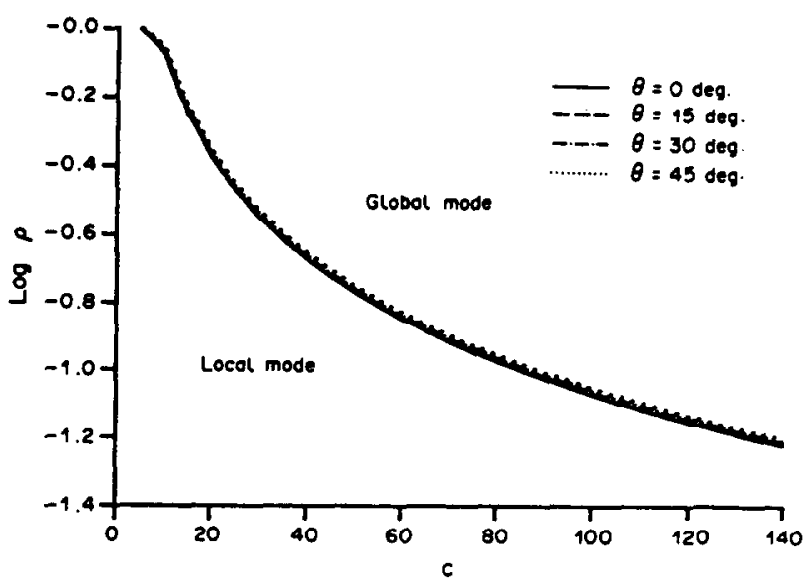

Fig. 9. Boundaries in the mass per unit area $\rho$ versus beam shape parameter $c$ separating the region where the critical mode is a local one from the region where the critical mode is a global one. Boundaries calculated for various values of the load angle $\theta$.

\section{CONCLUSIONS}

The purpose of the present work is the study of size effects in optimization problems involving microstructure, an issue of practical interest that has not been addressed thus far. One important physical mechanism on which attention is focussed in this study and which relates the size of the unit cell with the applied stresses is elastic buckling.

In order to avoid boundary effects, an infinite periodic structure is considered. Moreover, the structure's unit cell consists of axially compressed elastic beams thus greatly facilitating all the pertaining buckling load calculations that can be carried out analytically. By optimizing the aforedescribed periodic medium against buckling. i.e. by searching for the maximum possible minimal buckling load over a set of designs, one can come up with maximum allowable stresses for a given cell size or equivalently with minimum size requirements for a given stress state.

In addition, one can also identify the regions in the design space that correspond to a local or a global optimal buckling mode. When a global mode is the critical one, i.e. when the wavelength of the buckling mode is much larger than the cell size, the global instabilityunlike the local one-can be predicted by studying the homogenized properties of the structure. Consequently one can provide safe regions in the design space for which a homogenization theory can be employed. Such information is of significant help for microstructure related optimization analyses with respect to different criteria, for all the information they use are the homogenized properties of the solid without any idea about their range of validity.

The methodology presented here for the determination of the critical loads and modes is applicable to arbitrary periodic microstructures. The only difference with the general case is that the solution to the linearized stability equations with the $\omega_{x}$ dependent boundary conditions will require finite element techniques, thus making the optimization problem much more time consuming. In principle the local-global boundary in design space can also be found numerically.

The issue of microstructure size effects in optimal design is a very important one for it provides with realistic information about the proper size of the microstructures. The present analysis provides a methodology to design the microstructure safely against buckling. Important questions that still remain to be solved are the influence of the boundaries of the macroscopic structures or the study of other mechanisms that also determine size such as stress concentrations.

Acknowledgements-This work was supported in part by the Danish Technical Research Council, Grant Nos. 164246.M (MPB) and 16-4353.M (NT). Both authors are grateful to Prof. J. E. Taylor for helpful discussions and for his continuing encouragement during the course of this work. 


\section{REFERENCES}

Bendsøe. M. P. and Kikuchi. N. (1988). Generating optimal topologies in structural design using a homogenization method. Comput. Meth. Appl. Mech. Engng (to appear).

Bensousson, A., Lions, J. L. and Papanicolaou, G. (1978). Asymptotic Analysis for Periodic Structures. NorthHolland.

Boccardo, L. and Marcellini, P. (1976). Sulla convergenza delle soluzioni di disequazioni variazionali. Annali di Mat. Pura ed Appl. 110, 137-159.

Cheng, K. T. and Olhoff, N. (1982). Regularized formulation for the optimal design of axisymmetric plates. Int. J. Solids Siructures 18, 153-169.

Geymonat, P., Muller, S. and Triantafyllidis, N. (1989). Homogenization for Nonlinear Elastic Materials, Microbuckling and Loss of Rank One Ellipticity (submitted for publication).

Haug. E. J., Choi, K. K. and Komkov, V. (1986). Design Sensitivity Analysis of Structural Systems. Academic Press.

Kesavan, S. (1979). Homogenization of elliptic eigenvalue problems. Appl. Math. Optim. 5, 153-167 (part I) and 197-216 (part II).

Kohn, R. V. and Strang, G. (1986). Optimal design and relaxation of variational problems. Comm. Pure Appl. Math. 39, 1-25 (part I), 139-182 (Part II) and 353-377 (Part III).

Kohn, R. V. (1988). Recent Progress in the Mathematical Modeling of Composite Materials. Preprint, Courant Institute, NYU.

Liveseley, R. K. (1968). Matrix Methods in Structural Analysis. Pergamon Press.

Madsen, K. and Tingleff, O. (1986). Robust Structures for Non-linear Optimization. Report NI 86-01, Numerical Institute, Technical University of Denmark, DK-2800 Lyngby.

Mignot, F., Puel, J. P. and Suquet, P. M. (1980). Homogenization and bifurcation of perforated plates. Int. J. Engng Sci. 18, 409-414.

Muller, S (1987). Homogenization of nonconvex integral functionals and cellular elastic materials. Arch. Rat. Mech. Analysis 99, 189-212.

Ogden, R. W. (1984). Non Linear Elastic Deformations. John Wiley.

Olhoff, N. and Taylor, J. E. (1983). On structural optimization. J. Appl. Mech. 50, 1134-1151.

Sanchez-Palencia, E. (1980). Non-homogeneous media and vibration theory. Lecture Notes in Physics 127, Springer.

Suquet, P. (1981). Prevision par Homogeneisation du Flambement de Plaques Elastiques Renforcees. Rech. Aerosp. 99-107.

Triantafyllidis, N. and Maker B. (1985). On the comparison between microscopic and macroscopic instability mechanisms in a class of fiber reinforced composites. J. Appl. Mech. 52, 794-800.

\section{APPENDIX A. INCREMENTAL STIFFNESS MATRICES OF A PLANAR FRAME}

The derivation of the incremental stiffness matrices for a planar frame with axially prestressed members has already been presented in the literature and the interested reader is referred to the very readable book by Livesley (1968) for further details. For reasons of completeness of the presentation, however, all the appropriate formulas for the incremental stiffness matrices of the prestressed frame that were employed in Section 2 will be recorded here.

The starting point for the derivations is the potential energy for a beam of length $a$, cross sectional area $A$, moment of inertia $I$ and Young's modulus $E$ which is taken to be

$$
E=(1 / 2) \int_{0}^{a}\left\{E I\left(w_{x x}\right)^{2}+E A\left[u_{x}+(1 / 2)\left(w_{x}\right)^{2}\right]^{2}\right\} \mathrm{d} x+\text { Boundary Terms }
$$

where $u(x), w(x)$ are the axial and normal deflections of the beam. Assuming that the beam is subjected to end displacements and rotations:

$$
d_{1}=\left[u(0), w(0), w_{x}(0)\right], d_{2}=\left[u(a), w(a), w_{x}(a)\right]
$$

one can with the help of the equilibrium equations $\delta E=0$ express the potential energy in terms of the imposed end conditions. The incremental stiffness matrices relating the increments of the force vectors $t_{1}$ and $f_{2}$ at $x=0$ and $x=a$ respectively to the corresponding work conjugate increments of the displacement vectors $d_{1}$ and $d_{2}$, are given by:

$$
\mathrm{f}_{x}=\Sigma \mathbf{K}_{\alpha \beta} \dot{d}_{\beta}, \text { with } K_{\alpha \beta} \equiv \partial E / \partial \mathbf{d}_{x} \partial \mathbf{d}_{\beta}
$$

For the case of a beam prestressed by a compressive axial force $N$, the derivatives of the potential energy shown in (A2) evaluated at the (straight) principal solution in which $u(x)=-x N / E A+$ const, $w(x)=0$ give the following results: 
$\mathbf{K}_{11}=\left[\begin{array}{ccc}\frac{E A}{a} & 0 & 0 \\ 0 & \frac{12 E I}{a^{3}} h_{1} & \frac{6 E I}{a^{2}} h_{2} \\ 0 & \frac{6 E I}{a^{2}} h_{2} & \frac{4 E I}{a} h_{3}\end{array}\right], \quad \mathbf{K}_{22}=\left[\begin{array}{ccc}\frac{E A}{a} & 0 & 0 \\ 0 & \frac{12 E I}{a^{3}} h_{1}-\frac{6 E I}{a^{2}} h_{2} \\ 0 & -\frac{6 E I}{a^{2}} h_{2} & \frac{4 E I}{a} h_{3}\end{array}\right]$

$$
\mathbf{K}_{12}-\mathbf{K}_{21}^{T}=\left[\begin{array}{ccc}
-\frac{E A}{a} & 0 & 0 \\
0 & -\frac{12 E I}{a^{3}} h_{1} & \frac{6 E I}{a^{2}} h_{2} \\
0 & -\frac{6 E I}{a^{2}} h_{2} & \frac{2 E I}{a} h_{4}
\end{array}\right]
$$

where the coefficients $h_{1}, h_{2}, h_{3}, h_{4}$ depend on the axial force $N$ via:

$$
\begin{aligned}
h_{1} & \equiv(\sigma+\tau) / 6-\beta^{2} / 12, \quad h_{2} \equiv(\sigma+\tau) / 6, \quad h_{3} \equiv \sigma / 4, \quad h_{4} \equiv \tau / 2 \\
\sigma & \equiv \beta(\sin \beta-\beta \cos \beta) /[2(1-\cos \beta)-\beta \sin \beta] \\
\tau & \equiv \beta(\beta-\sin \beta) /[2(1-\cos \beta)-\beta \sin \beta] \\
\beta^{2} & \equiv N a^{2} / E I .
\end{aligned}
$$

The above results hold for the case of the $x_{1}$ axis aligned with the axis of the beam. If, as in the case of the application considered in Section 2, the axis of the member in question forms an angle $\psi$ with respect to the $x_{1}$ axis (recall that all the nodal forces and displacements of the entire structure are resolved with respect to the same frame $x_{1}, x_{2}$ ) then the $K_{x \beta}$ in (A2) are replaced by:

$$
\begin{gathered}
\left(\mathbf{K}_{x \beta}\right)_{\text {new }}=\mathbf{T}\left(\mathbf{K}_{x \beta}\right)_{\text {ddd }} \mathbf{T}^{\tau} \\
\mathbf{T}=\left[\begin{array}{ccc}
\cos \psi & -\sin \psi & 0 \\
\sin \psi & \cos \psi & 0 \\
0 & 0 & 1
\end{array}\right]
\end{gathered}
$$

where the $\left(\mathbf{K}_{\alpha \beta}\right)_{\text {od }}$ are the stiffnesses with respect to the local system of the member in question given by (A3). 